\title{
Installation Des Colons Agricoles Dans Le Terroir Du Confluent Ouème-Beffa À Ouèsse Au Centre Du Bénin: Impacts Socio-Economique Et Environnemental
}

\author{
Alphonse A. Alomasso \\ Laboratoire de Climatologie \& Ethnoclimatologie Tropicales, \\ Université de Parakou, Benin \\ Vodounou Jean Bosco \\ Marcel Adigbegnon \\ Département de Géographie et Aménagement du Territoire, \\ Université de Parakou, Bénin
}

Doi: 10.19044/esj.2018.v14n27p40 URL:http://dx.doi.org/10.19044/esj.2018.v14n27p40

\begin{abstract}
The depletion of natural resources has resulted in a change in farming systems and the change in social relations that have long characterized African societies. For example, slash-and-burn agriculture that has characterized cropping systems in these countries has shown its limits in some areas. In Benin it took new forms resulting in the abandonment of certain soils for the benefit of others: the migration of peasants. This phenomenon has become so widespread today that pressure has been felt in previously vacant areas, leading to many conflicts and tensions between indigenous and immigrants, creating a degree of land insecurity for indigenous people that does not allow them to do so. to adopt sustainable practices for a good use of the land resource. The results obtained were possible thanks to the fieldwork and through the deductive method and descriptive, analytical and cartographic approach. This study has identified the socio-economic and environmental impacts of the massive arrival of agricultural settlers in the land of the confluence Oumé-Beffa, and identify the constraints related to the colonization of agricultural land by migrants and the strategies that they develop to better valorise their contracts in order to propose adapted systems for a sustainable management of the fertility of the lands in the conflict of Oueme-beffa in Ouessè.
\end{abstract}

Keywords: Agricultural colonists, exhaustion, cropping systems, migration 


\section{Résumé}

L'épuisement des ressources naturelles s'est traduit par une modification des systèmes de culture et le changement des rapports sociaux qui ont longtemps caractérisé les sociétés africaines. Ainsi l'agriculture itinérante sur brûlis qui a caractérisé les systèmes de cultures dans ces pays a montré ses limites dans certaines zones. Au Bénin elle a pris de nouvelles formes se traduisant par l'abandon de certains terroirs au profit d'autres : la migration des paysans. Ce phénomène a pris de nos jours une telle ampleur qu'une pression sur les ressources s'est fait sentir dans les zones auparavant vacantes, amenant de nombreux conflits et tensions entre autochtones et immigrants engendrant une certaine insécurité foncière pour les autochtones qui ne leur permet d'adopter des pratiques durables pour une bonne utilisation de la ressource terre. Les résultats obtenus a été possible grâce aux travaux de terrain et à travers la méthode déductive et approche descriptive, analytique et cartographique. La présente étude a permis de dégager les impacts socioéconomique et environnemental de l'arrivée massive des colons agricoles dans le terroir du confluent Oumé-Beffa, et l'identifier les contraintes liées à la colonisation des terres agricoles par les migrants et les stratégies qu'ils développent pour mieux valoriser leurs contrats afin de proposer des systèmes adaptés pour une gestion durable de la fertilité des terres dans le conflient de $1^{\prime}$ Oueme-beffa à Ouessè.

Mots clés: Colons agricoles, épuisement, systèmes de cultures, migration

\section{Introduction}

Dans les pays en voie de développement en général, et au Bénin en particulier, l'agriculture constitue la base de l'économie et la principale source de revenu en raison notamment de sa contribution au PIB (40\%) et de la part de la population qu'elle mobilise environ 80\% (Alomasso, 2009). Malgré cette prépondérance du secteur primaire, l'agriculture du Bénin demeure une agriculture minière, itinérante sur brûlis, fortement tributaire des saisons et d'autres contraintes naturelles et utilisant jusqu'à nos jours des outils rudimentaires, archaïques. Ainsi, cette situation augure de faibles performances économiques de cet état ainsi que leur niveau de développement (Kazenwadel, Edja, 1999). En effet, étant donné que l'évolution des techniques agricoles adaptées économiquement rentables et financièrement accessibles) ne suit pas l'accroissement galopant des populations et face à cette insuffisance d'emplois alternatifs dans les autres secteurs, la population pour satisfaire ses besoins vitaux, se rabat sur les ressources naturelles (sols, forêts et eaux) à moindre coût financier (Biaou, 1996). 
Ainsi la pression démographique qui en résulte laisse cette population dans la misère qui l'oblige ; à l'exode et à la migration av la politique vise à garantir à cette population rurale, la satisfaction de ses besoins fondamentaux et à maintenir le potentiel des ressources déjà peu disponibles. Ainsi la présente étude tente de faire une analyse de l'utilisation de la ressource terre dans une zone de colonisation récente en proie à une pression de plus en plus forte liée au phénomène d'immigration avec sans soucis de préservation de ressources naturelles. En effet, la migration des populations vers les régions disposant encore de terres cultivables a pris de nos jours une ampleur importante entraînant une insécurité foncière en raison des contrats d'utilisation des terres de plus en plus défavorisant aux immigrants alors qu'il est reconnu que la sécurité foncière est un facteur clé de la gestion des ressources naturelles, en général, de la fertilité des sols et l'adoption des systèmes agraires durables en particulier (Biaou,1991\& 1996; Neef,1997; Hounkpodote, 2001; Gerder-lig et petermann, 2002). La production agricole dans notre secteur d'étude situé dans la $5^{\text {ème }}$ zone agro-écologique du Bénin.

\section{Données et méthodes}

\subsection{Caractéristiques physico-géographiques du secteur d'étude}

La commune de Ouessè est située au centre du Bénin entre $8^{\circ} 10^{\prime}$ et $8^{\circ} 45^{\prime}$ de latitude Nord et $2^{\circ} 10^{\prime}$ et $2^{\circ} 45^{\prime}$ de longitude Est (figure 1) et couvre une superficie d'environ $3200 \mathrm{~km}^{2}$, soit 2,56 \% du territoire national. Du fait de sa position géographique, elle jouit d'un climat tropical intermédiaire dit de climat de transition entre les climats guinéens au Sud et soudanien au Nord. Selon INSAE (2016), sa population est estimée à 142017 habitantsen 2013, donnant une densité de 44 habitants $/ \mathrm{km}^{2}$. Cette croissance démographique a conduit à une utilisation non contrôlé des terres agricoles ce qui a eu pour conséquences leur épuisement (Adigbenon et al., 2017). Ces conséquences sont à l'origine des migrations des agriculteurs observés. 


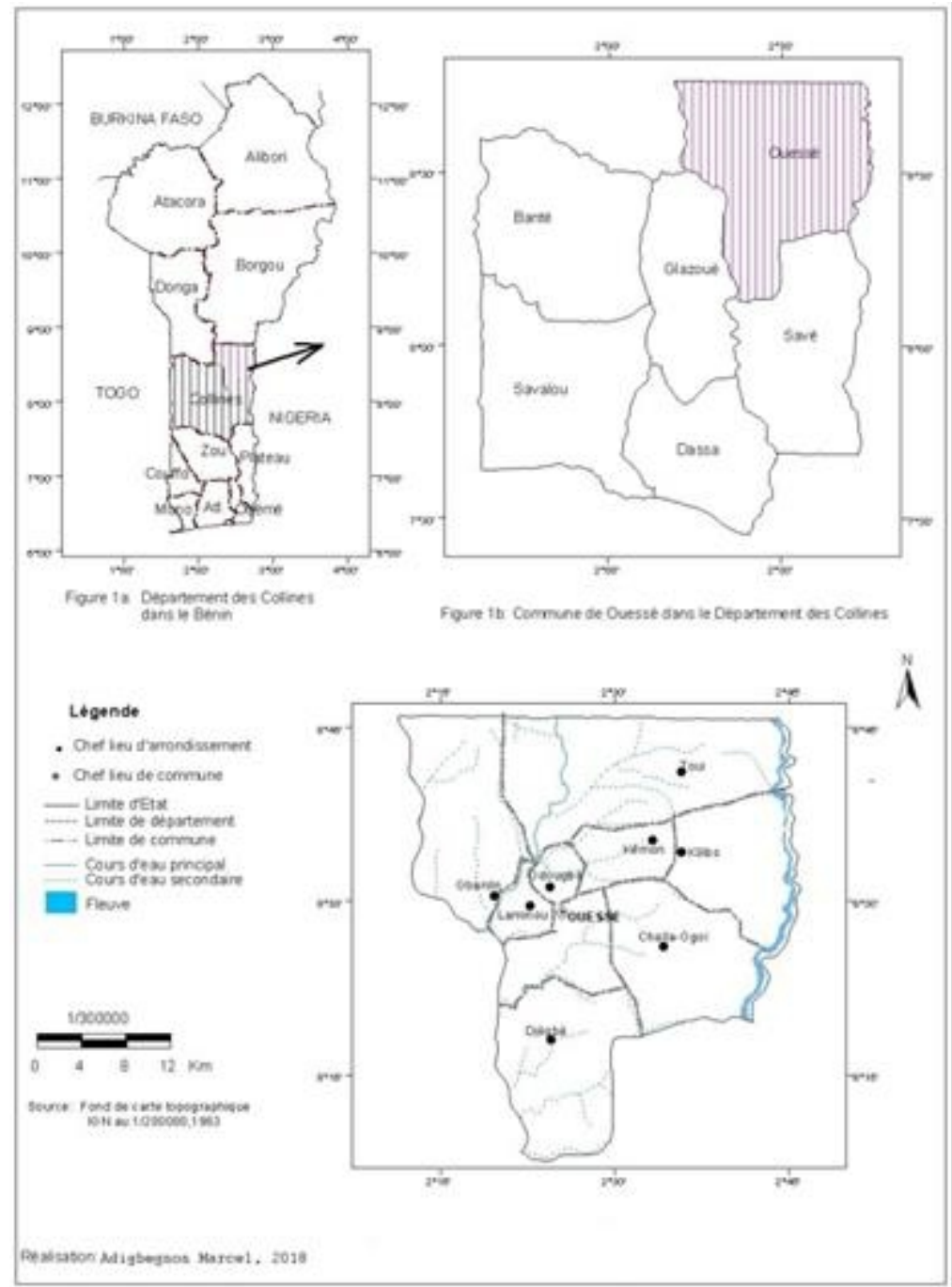

Figure 1: Situation géographique de la commune de Savè

Située au centre du Bénin, la commune de jouit d'un climat tropical intermédiaire dite de climat de transition entre les climats Guinéen au Sud et Soudanien au Nord (Adigbegnon et al., 2017). La pluviométrie et la température moyennes annuelles sont respectivement de 1058,8 $\pm 193,1 \mathrm{~mm}$ et de $27,7 \pm 1,5^{\circ}$ Csur la période 1971-2015. Selon Alomasso (2009), le Relief de la Commune de Ouèssè est un plateau vallonné dominé par quelques collines localisées à Kokoro, Challagoi, Yaoui, Kêmon et Kilibo d'altitude variant de 200 à $350 \mathrm{~m}$ et avec des pentes de 3 à $6 \%$. Les plateaux représentent $75 \%$ de la région, les collines $15 \%$ (surtout concentrées dans l'Est) et les bas-fonds $10 \%$ (Aho, 1994). La végétation a un attrait forestier et savanicole diversifié. Les activités économiques dans le secteur d'étude sont l'agriculture, l'élevage 
et l'exploitation forestière mais l'agriculture à elle seule concentre plus $90 \%$ de la population (Akpassonou et Houindo, 1994). Avec des potentialités en terres agricoles de 1 'ordre de $25,99 \%$, ces terres sont en train de s'épuiser ce qui à créer les mouvements migratoires observé à Ouessè.

\subsection{Données}

Les statistiques démographiques, fournies par l'Institut National de la Statistique et de l'économie (INSAE) pour les recensements généraux ont servi aussi de base à cette étude. Les données des statistiques agricoles telles que les superficies emblavées pour la production ont été collectées au CARDER/Ouessè pour mesurer la réduction ou non de la production agricole. De plus, les données d'occupation du sol sont prises en compte dans l'analyse de l'épuisement des sols. Les données sur les mouvements migratoires à Ouessè ont été obtenues à partir des statistiques de l'INSAE. Ces données ont été complétées par celles du terrain. Elles données sont celles liées aux taux d'accroissement de population $\mathrm{t}$ aux mouvements de la population. Sur carte de flux de migration, Les mouvements sont représentés sur la carte par flèches en polygone d'épaisseur variant, proportionnelles (Arouna et Zakari, 2016) au des migrants.

\subsection{Analyse des mouvements d'installation des colons agricoles}

A cause de l'indisponibilité des données aux mouvements d'installation des colons agricoles dans le secteur d'étude, il a été pris en compte les données quantitatives et qualitatives issues des enquêtes de terrain ont été utilisées. Les avis recueillis par entretien direct ont permis d'identifier les lieux de destination des migrants agricoles dont le point de départ est Ouessè et les principales raisons de leur déplacement. Les réponses ont été analysées avec une certaine méfiance au regard du cadre théorique pour détecter les points à améliorer pour mieux réussir la lutte gérer le poids des réfugier écologiques.

\section{Résultats}

\subsection{Processus d'installation des colons agricole}

\subsubsection{Caractéristiques de l'immigration dans le confluent Ouémé-Beffa.}

Les mouvements d'immigration observés dans la Commune de Ouèssè en l'occurrence dans le terroir du confluent concernent la colonisation agricole, la transhumance, et ouvriers agricoles. Cette étude s'est intéressée aux colons agricoles. A la différence des ouvriers agricoles qui viennent pour la circonstance, les colons s'installent pour une longue durée et travaillent pour leur propre compte. Quant aux ouvriers agricoles, ils signent un contrat d'un an renouvelable. Cette migration affecte le plus souvent les populations d'une même origine ethnique (Les fon de Finagnon à Vossa et Berba de la ferme 
Moukoun d'Idadjo). La figure présente l'architecture des colons agricoles dans le secteur d'étude.

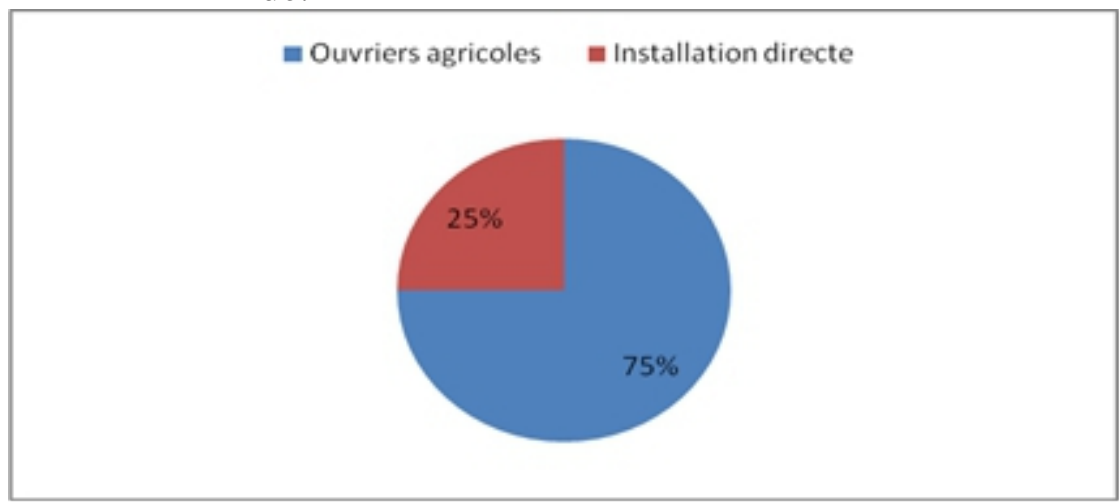

Figure2 : Architecture d'installation des colons agricoles

Les résultats des enquêtes indiquent $75 \%$ de ces colons se constituent d'abord en ouvriers agricoles avant de s'installer définitivement. Mais ils peuvent également s'installer directement 25\%. Quant aux ouvriers agricoles à peine $17 \%$ des autochtones parviennent à y recourir. Les colons choisissent leur site d'accueil en fonction les opportunités et la répartition des anciens venus sur le terrain. Ainsi le tableau suivant donne une idée de la dominance par ferme.

Tableaux I : Choix de site des colons dans les quatre villages d'étude

\begin{tabular}{|c|c|c|c|}
\hline Village & $\begin{array}{l}\text { Ethnies } \\
\text { dominantes }\end{array}$ & Village du départ & Sites d'accueils \\
\hline Vossa & Fon & $\begin{array}{l}\text { Kpozoun } \\
\text { Tindji } \\
\text { Tanguiéta, } \\
\text { GouandéDassari } \\
\text { Matéri, Tantéka, }\end{array}$ & $\begin{array}{l}\text {-Ferme finagnon } \\
\text {-Ferme Wokpa } \\
\text {-Ferme pendjari } \\
\text { Ferme Tchatchadji }\end{array}$ \\
\hline Gbanlin & Berba & Tangéuita, Gouandé & $\begin{array}{l}\text {-Ferme mouadjo } \\
\text {-Saagoudji }\end{array}$ \\
\hline $\begin{array}{l}\text { Tosso } \\
\text { Idadjo }\end{array}$ & $\begin{array}{l}\text { Bètammaribè } \\
\text { Berba }\end{array}$ & $\begin{array}{l}\text { Dassari } \\
\text { Matéri, Tantéka, Gouandé, } \\
\text { Kandihoun }\end{array}$ & $\begin{array}{l}\text { Sinlindji } \\
\text { Mounkoun }\end{array}$ \\
\hline
\end{tabular}

Source : Résultat d'enquête de terrain, Juillet 2008

\subsubsection{Tendances de l'immigration dans le secteur du Confluent Ouémé- Beffa}

Les différents groupes sociaux des colons viennent chaque année en grossir le groupe des anciens. Les Fon venus du plateau d'Abomey sont les groupes sociaux dominant de tous les colons de notre secteur d'étude soit un pourcentage de (35\%) ensuite viennent les Berba qui représentent les $25 \%$. 
Parmi les causes qui expliquent l'immigration des populations dans le confluent Ouemé-Beffa on peut citer les contraintes du milieu de départ et les atouts offerts par le milieu d'accueil.

\subsubsection{Contraintes liés aux mouvements migratoires}

Elles sont relatives aux conditions spécifiques qui prévalent dans les régions de départ. Parmi elles on peut citer les causes financières, les causes sociologiques, les contraintes du milieu naturel (figure ) et les facilités offertes par le milieu d'accueil.

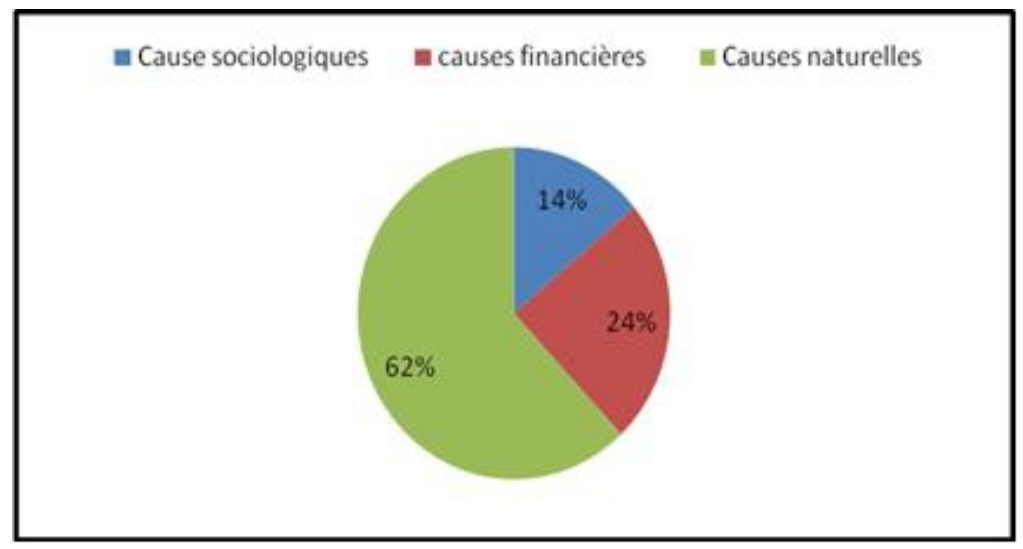

Figure3 : Répartition des raisons de mouvements des agriculteurs

De l'analyse de la figure (), il ressort que les causes financières représentent $24 \%$ des cas, les causes de l'immigration des populations vers la Commune de Ouèssè. Ce qui n'est rien d'autre qu'une expression de volonté d'indépendance qui ne peut être pleinement acquise sans un minimum de sécurité matérielle qu'il pense acquérir à l'étranger. A cela s'ajoutent les causes sociologiques.Elles viennent après les causes financières et représentent pour $14 \%$ les raisons qui poussent les colons, surtout les plus jeunes, à émigrer de leur milieu d'origine. Parmi elles, on distingue les contraintes familiales liées aux différents liens de soumission des jeunes à leurs parents et les causes psychologiques liées à l'effet de démonstration des anciens migrants qui reviennent d'exil et le besoin de s'ouvrir au monde extérieur.Ainsi, l'exode rural en général et la colonisation agricole en particulier deviennent une sorte d'initiation à l'autre monde que les jeunes doivent faire pour s'affirmer dans leurs villages. De cette même figure il faut noter qu'il y a des contraintes naturelles avec $62 \%$, elles concernent principalement les contraintes climatiques, l'épuisement des sols, le morcellement excessif des terres et le développement des cultures de rente. Cependant, les problèmes d'enclavement : ils rendent le travail agricole mal payé, faute de routes de qualité pour convoyer les produits vers les marchés de regroupement où les conditions de vente sont bien meilleures. A toutes ces 
contraintes, il faut ajouter la forte concentration démographique qui caractérise ces Communes.

\subsubsection{Facilités offertes par le milieu d'accueil}

La Commune Ouèssè à l'instar de Savè est une région tampon entre le centre et le Nord- Bénin. Cette situation fait d'elle un pôle d'attraction aussi bien pour les populations du sud que du Nord. Ce qui traduit la forte concentration démographique. En effet,les facilités offertes par le milieu naturel sont liées à la faible densité démographique qui encourage lesimmigrations vers le milieu ; la souplesse du régime foncier à travers des conditions simples d'accès a la terre ; l'existence de nombreux points d'eau et de pâturages et ceci grâce à l'appui des bailleurs comme Pays-Bas, AFD et UNICEF dans l'implantation des points d'eau, notamment les forages pompes manuelles (FPM) dans le cadre de la décentralisation. Il est remarqué que 75\% des fermes et hameaux parcourus ont déjà bénéficié de ces ouvrages. Formant de petites agglomérations ces colons agricoles ont bénéficié de l'implantation des écoles dans quatre fermes (Wokpa, Mouadjo, Gambari, Wodji). A cela il faut ajouter les besoins des autochtones en mains d'œuvres salariées et la situation charnière de la commune.

\subsection{Colonisation et mutation agricole dans le secteur}

\subsubsection{Colonisation et production agricole dans le confluent de Ouémé- Gbeffa}

L'évolution de la production agricole dans un milieu donné n'est pas seulement le fait de l'abondance des pluies et de sa répartition spatiale, de la qualité des sols, d'un meilleur encadrement des paysans et du dynamisme des marchés agricoles. Elle implique aussi l'abondance et le dynamisme de la main-d'œuvre agricole. C'est pourquoi l'analyse de cette question est délicate.Ce paragraphe examinera en plus de l'évolution de la production agricole et de sa répartition spatiale, la place qu'occupent les colons agricoles dans cette production.

\subsubsection{Evolution de la production agricole}

L'activité humaine responsable de la dégradation des terres dans la région de Ouessè est la production agricole qui occupe entre $80 \%$ (Akpaki, 2007) et $90 \%$ (Akpassonou et Houndo, 1994). Pour $62 \%$ des personnes enquêtées, il faut étendre les superficies avant que le couvert végétal ne soit touché par l'effet de la production agricole. Cette production est non seulement le fruit du travail des autochtones mais aussi celui des colons agricoles. Ainsi, lors de la compagne 2004-2005 2006-2007 pour la production du manioc par exemple Ouessè a occupé le premier rang avec une production respective de $39,67 \%$ et $34,35 \%$ du total au niveau départemental. 
Les paysans de la Commune de Ouèssè en l'occurrence ceux du secteur d'étude cultivent une gamme variée de produits. La figure présente l'évolution de la production agricole et des superficies emblavées par les spéculations de la région de Ouessè.

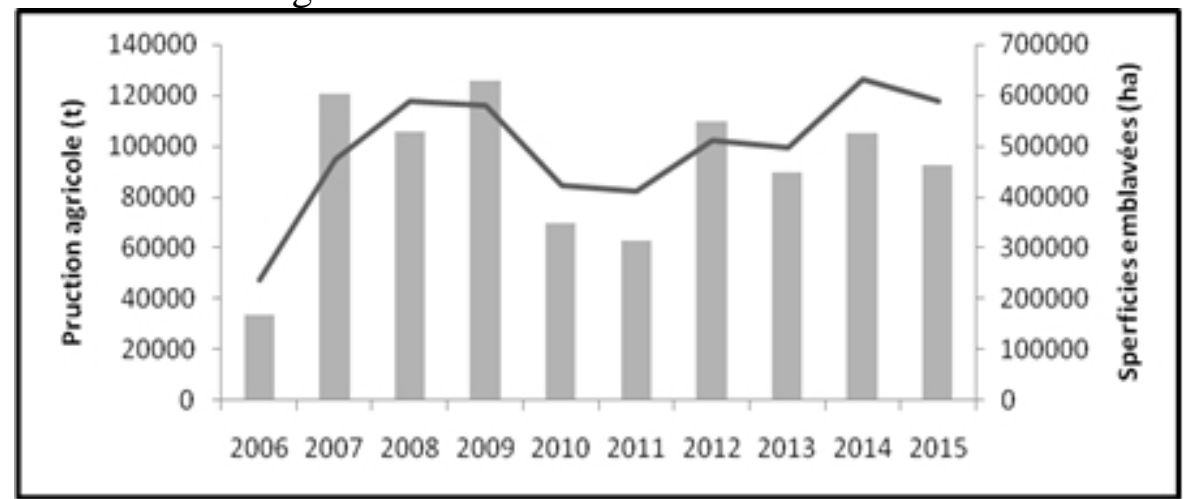

Figure 4 : Evolution de la production et des superficies emblavées entre 2006-2015

La production agricole et les superficies ont connues une variation au cours de la période 2006 à 2015 dans la région de Ouessè. Cependant, les vastes superficies observées dans la région de Ouessè entre 2006 et 2015 ont conduit à une altération de la végétation et une fois la végétation est détruite, le sol est exposé à l'érosion(photo 1).
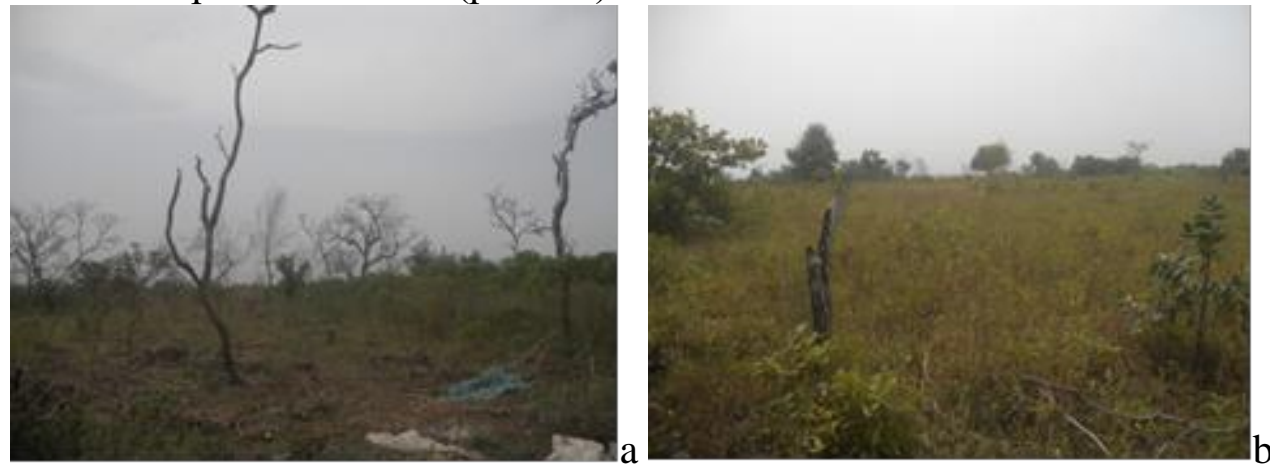

Photo 3 : Couvert végétal brûlé dans un champ de manioc à Kilibodji (Ouessè) et terre infertile Moumadjamè (Ouessè)

Prise de vue :ALOMASSO .A., novembre 2016

La production agricole sur un sol pendant plusieurs années successives réduit sa fertilité par conséquent le déplacement sur un autre lieu s'impose. C'est ce qui explique la réduction des superficies emblavées de 16,84\% la baisse de la production agricole de l'ordre 14,45\% pendant les années 2010 et 2011. En effet, les statistiques agricoles sont passées respectivement de 169093 tonnes à 628853 tonneset de 47196 hectares à 116096 hectares entre 2006 et 2009. Ce qui explique l'abondance d'une main d'œuvre qui peut se traduire soit par une population moins dynamique soit par l'immigration plus 
accrue des agriculteurs venus du Nord-ouest du Bénin (25\%) et ceux du département du Zou(35\%) (Alomasso, 2009). Cet état de chose conduit à la détérioration progressive de la couverture végétale. Les déplacements des populations surtout à la recherche de terres plus fertiles accroissent la dynamique de l'occupation du sol dans le secteur d'étude.

Leur poids démographique, leur talent de cultivateur firent d'eux les grands producteurs du milieu.

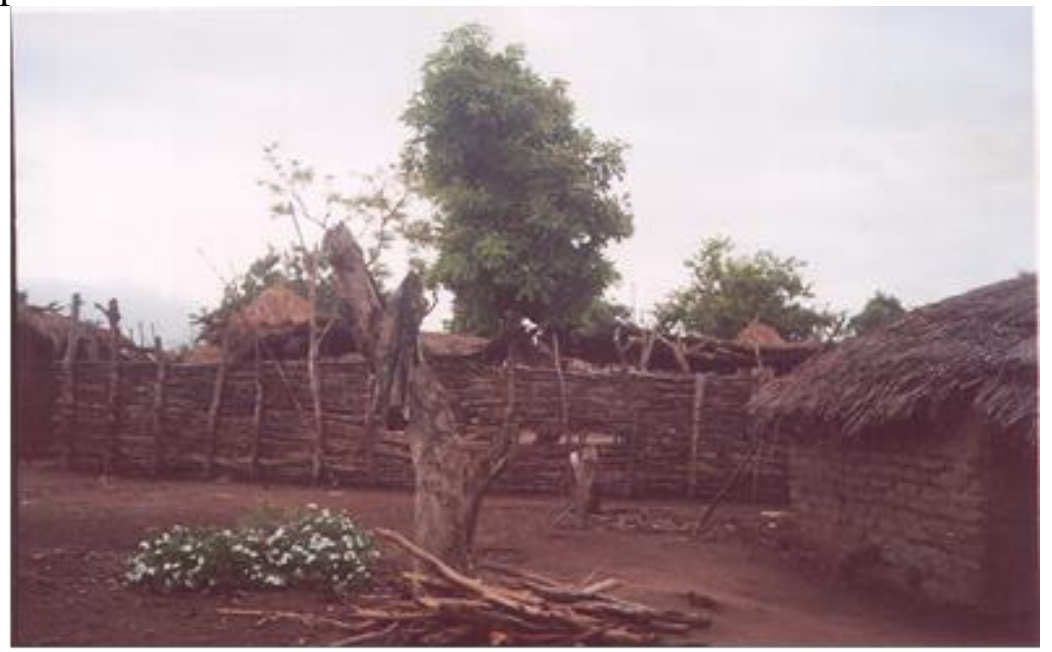

Photo 4 : Habitation type des Berba de Wodji

Cliché : ALOMASSO ; Juin 2016

\section{Conclusion}

En abordant cette étude la préoccupation est d'analyser l'impact de la colonisation agricole sur le terroir du confluent Ouémé-Beffa dans la commune de Ouèssè au regard de l'évolution que ce phénomène a pris depuis les années 1990. En effet, depuis les indépendances l'installation des colons agricoles a engendré de profondes mutations dans le domaine agricole et celui de la conservation de l'environnement.

Au terme de cette étude il ressort que le phénomène est devenu plus important dans la mesure où les colons deviennent de plus en plus nombreux dépassant en nombre les autochtones par endroits. C'est le cas de la ferme Wokpa en comparaison avec le village Tosso. L'ampleur du phénomène de colonisation agricole se traduit par la prolifération des habitats des colons.

Ce facteur de colonisation a contribué à la modification du milieu. Laquelle modification peut être appréciée à plusieurs niveaux. Au niveau des activités rurales : deux points méritent d'être souligné. L'importance de la production qui contraste avec celle à laquelle on pouvait s'attendre en tenant compte de la forte émigration qui touche les populations autochtones. Le dynamisme des marchés liés aux talents des colons agricoles. Ces derniers fournissent la plus grosse part des excédents commercialisables La 
transformation du paysage rural dont les formes les plus visibles sont l'habitat rural, cas de la ferme Wokpa. Une autre forme de la modification du paysage rural est celle relative à la dégradation du couvert écologique liée à la transhumance fulani et aux systèmes de culture des colons agricoles.

\section{References:}

1. Adigbegnon M., Bamisso R., Totin V. S. H., Amoussou E., Houndenou C., Boko M., (2017). Facteurs climatiques et anthropiques de la désertification dans le centre du Benin (commune de Ouessè). Dossiers Société Environnement Développement, La revue scientifique-les cahiers du CBRST, N¹1, Cotonou, Bénin, pp. 362390.

2. Akpaki J., (2007). L'avenir du pastoralisme face aux mutations socioéconomiques au centre du Bénin : Cas du secteur Tchaourou/Ouessè, Rapport technique, Cotonou, Bénin, $15 \mathrm{p}$.

3. Akpassonou C. P., Houindo Y. M., (1994). Etude de la dynamique des paysages en milieux Maxi, sous-préfecture de Ouessè : Influence de l'homme sur le milieu. Mémoire de maitrise, Université d'AbomeyCalavi, Cotonou, Bénin, 103 p.

4. Alomasso A. A., (2009). L'installation des colons agricoles dans le terroir du confluent oueme-beffa a Ouesse : Impacts socioéconomique et environnemental. Mémoire de maîtrise Géographie Physique, Département de Géographie et Aménagement du Territoire, FLASH, Université d'Abomey-Calavi, Cotonou, Bénin, 96 p.

5. Arouna O., Zakari S., (2016). Mobilités et migrations des populations en Afrique de l'Ouest. Revue bibliographique et cartographique, Observatoire du Sahara et du Sahel (OSS), Université Polytechnique d'Abomey, Laboratoire de Cartographie d'Abomey-Calavi, Cotonou, Bénin, 48 p.

6. Zakari S., Agossou B., Tente H., Imorou I. T., Yabi I., Afouda F., N'bessa B., (2015). Variabilité hydropluviométrique et dynamique de l'occupation des terres dans le basin de la Sota à l'exutoire de Coubéri au Bénin (Afrique de l'Ouest). International Journal of Innovation and Applied Studies ISSN 2028-9324 Vol. 13 No. 2 Oct. 2015, pp. 235250 . 